\title{
Decoro urbano. Apuntes de ida y vuelta sobre Arte, Espacio Público y ambientes urbanos de calidad
}

\section{Urban decor Notes of round trip on Art, Public Space and quality urban environments}

DOI: 10.17981/mod.arq.cuc.19.1.2017.01

Artículo. Fecha de recepción: 25/08/2017 Fecha de aceptación: 27/10/2017

\section{Antoni Remesar}

CR POLIS. CRIT Research Group. Universitat de Barcelona (España)

aremesar@ub.edu.co

Para citar este artículo:

Remesar, A. (2017). Decoro urbano. Apuntes de ida y vuelta sobre Arte, Espacio Público y ambientes urbanos de calidad. MODULO ARQUITECTURA-CUC, vol. 19, no. 1, pp. 9-20. DOI: 10.17981/mod.arq.cuc.19.1.2017.01

\section{Resumen}

Desde el Renacimiento, hacer una ciudad hermosa es una cuestión recurrente en la teoría y práctica urbana occidental. Sobre el trasfondo de esta problemática se puede ubicar el gran tema del decoro urbano, "el 'ajuste' de los medios expresables al contenido expresado" (Tafuri, 1968). Los tratadistas clásicos ${ }^{1}$ declararon las ideas principales para un entorno urbano organizado y hermoso. Otra manifestación de estas ideas fue el uso monumental de la escultura en lugares públicos situando una estatua o un obelisco en el centro de una plaza, una tradición que se adoptó ampliamente después del siglo XVI.

Palabras clave: Decoro Urbano, Espacio público, Imagen de la ciudad, Arte público, Arte Civico, Diseño civico.

\section{Abstract}

Since the Renaissance, making a beautiful city is a recurrent issue in Western urban theory and practice. Against the background of this problem can be located the great theme of urban decorum, "the 'adjustment' of the means expressible to expressed content" (Tafuri, 1968). The classical writers declared the main ideas for an organized and beautiful urban environment. Another manifestation of these ideas was the monumental use of sculpture in public places by placing a statue or an obelisk in the center of a plaza, a tradition that was widely adopted after the sixteenth century.

Keywords: Decoro Urbano, Public space, City image, Public art, Civic art, Civic design.

1 "La calle que discurre dentro de la ciudad estará decorada con dos pórticos de idéntico diseño, y las casas estarán alineadas a ambos lados e iguales en altura, además de que debe estar absolutamente limpia y bien pavimentada. Sin embargo, las partes de la calle donde se aplicará la ornamentación adecuada son las siguientes: el puente que cruza la plaza, el lugar destinado a espectáculos. La plaza resultará de un cruce más ancho; y el espacio destinado a espectáculos no es más que una plaza rodeada de graderías" (Alberti, 1992). 
Si bien no es el primero, se puede considerar como paradigmático el diseño de la Plaza del Capitolio² (Campidoglio) de Miguel Ángel en el Capitolio de Roma, que se le encargó diseñar en 1537.

\footnotetext{
2 "Let me finish with one example. It is a tragic example. speak of the Capitol at Rome of Michelangelo. The area Capitolina occupies one of the hill top sites of the ancient city of Rome. It is composed of a complex of the square itsel (which is not a real square but more of a trapezoid); a broad ramped stairway (the Cordinata); and three buildings (the Senatorial Palace or town hall in the background, the Palazzo dei Conservatori on the right and the Capitolina Museum on the left). The architectural composition of the Capitol can be rapidly summarised as a comprehensive development in depth: piazza, stairway, and the visual relation with the old medieval city of Rome. In 1530, the city-republic of Florence lost its independence to the Medici despot, Cosimo the First. As you will know, Michelangelo came from an old Florentine family and, in 1534, he left Florence forever and spent the remaining thirty years of his life in voluntary exile in Rome. Here he gave concrete reality to what he had derived from his youthful democratic experiences in Florence. Here, in the Rome of the Counter-Reformation - a Rome in which there was no freedom and no democracy! Michelangelo's Capitola very perfect expression of the Core - was a symbol of the vanished liberties of the medieval city-republic that he held in his heart. It was, at the same time, a memorial to the tragic dreams of its creator. The lack of imagination usually shown today (though there are a few exceptions) in our attempts to devise new city centres - new city Cores - is invariably excused on the ground that we no longer have a way of life that it is possible to express. What Michelangelo has mirrored in his Area Capitolina is the baffling irrationality of historic events and the enigmatic omission of any direct relation between effect and cause. Once more we realise that a great artist is able to create the artistic form for a phase of future social development, long before that phase has begun to take tangible shape. This is our task today" (Giedion, 1952) o "this square, sculpture was placed next to buildings, working closely with, or as part of, buildings, leaving the centre of public spaces open for public use. Michelangelo, who was predominantly a sculptor himself, gave the centre of the square to a statue of Marcus Aurelius, the only equestrian statue to have survived from ancient Rome. This central place was emphasized by placing the statue at the centre of an ova pattern on the floor, and on the main axis of the square, which was marked by the stairs leading from the bottom of the hill to the square. This was the first monumental square of its kind paving the way for the Baroque squares that were created afterwards" (Mandanipour, 2007, p. 46).
}

No se pretende aquí rastrear la historia de la noción de Decorum urbano ${ }^{3}$. En general, el decoro se refiere a la idoneidad de un diseño. En el pasado, los diseñadores tuvieron que articular el significado de un edificio, que se define en términos de uso, condición social y la ubicación física. El decoro arquitectónico insistió en que un diseño debería estar de acuerdo con su propósito y adaptarse adecuadamente a su audiencia, es decir, a otros edificios y al público en general. "El decoro era por lo tanto una característica central de una idea más amplia de elocuencia cívica. El decoro impregnaba la teoría arquitectónica y urbana antes del siglo XIX" (Kohane \& Hill, 2001). En cualquier caso, la noción de decoro ha persistido a lo largo de los siglos, pero tomando diferentes significados, de los que se iniciará una primera exploración.

\footnotetext{
3 "Decorum in Western architectural theory derives from the treatises of Vitruvius (On Architecture) and Alberti (De re aedificatoria, 1485). In Vitruvius, appropriateness (decor) binds form to function, so that the siting of a building, its approaches, aspect and choice of order are determined by its purpose. Alberti amplifies Vitruvius's concern with fitting dignity (dignitas), introduces the term concinnitas (from which the dignity derives) and makes the architect's judgement of decorum so decisive that it determines even placement of the altar in churches. Of later theorists, Nicolas Le Camus de Mezières is important for the idea of appropriate architectural 'character' (caractère), as is A. W. N. Pugin and his 'Ecclesiological' followers, who justified a Gothic Revival style on grounds of fitness-for-purpose. This concept of decorum formed a theoretical basis for the Arts and crafts movement in Great Britain and France initially, then elsewhere. Indeed, the theory of Functionalism both in architecture and in 'craft' is tied to fitness-for-purpose and therefore to decorum" (Gaston, 2014).
} 


\section{Art URBAin}

Arte urbano $^{4}$ (Art Urbain) significa construir y planificar el espacio de las ciudades, tal como se teorizó desde el Quattrocento y, poco a poco, puesto en práctica durante el Renacimiento, la edad clásica y el período neoclásico.

El arte urbano introdujo en las ciudades occidentales la proporción, la regularidad, la simetría, la perspectiva, aplicándolos a las calles, plazas, edificios, y también al tratamiento de sus relaciones y sus elementos de conexión (arcadas, columnatas, puertas, arcos, jardines, obeliscos, fuentes, estatuas, etc.) (Choay, 1998).

\footnotetext{
4 Aquí aparece una primera disquisición de idioma. Actualmente el concepto francés "art urbain" corresponde al "diseño urbano" anglosajón, como se muestra en algunos documentos del Consejo Europeo de Urbanistas (Consejo Europeo de Urbanistas-CEU, 1998). Sin embargo, en este punto del artículo, preferimos usar el concepto de arte urbano ya que el significado expresado por Choay se refiere a la composición urbana, es decir, el conjunto de procedimientos figurativos, en dos o tres dimensiones, planteados para resolver un proyecto urbano desde una cierta estética. J. L. Sert (Sert, 1957) introdujo el concepto de diseño urbano, como una nueva disciplina, en 1956 durante una conferencia internacional en la Graduate School of Design de Harvard. Louis Mumford, Jane Jacobs, Victor Gruen, Edmund Bacon, Garrett Eckbo y Hideo Sasaki, entre otros, asistieron a esta conferencia. "In the decade preceding that event, urban planning had become increasingly less focused on the physical organization of the city, and had established its own independent academic and professional territory based on the methods of social science. The more comprehensive practice of "urbanism," at that time still dominant in Europe and Asia, was replaced in the United States by a dual structure that effectively disengaged the concerns of urban planning from those of architecture. At the Harvard conference, Sert announced urban design as a new academic field, which he defined as 'the part of planning concerned with the physical form of the city'. He added that the urban designer must firs believe in cities, their importance and their value to human progress and culture. Thus, academic urban design began with two agendas, one professionally oriented, the other culturally based" (Kahn, 2002)
}

En este sentido, a fines del siglo XVIII, Antoine Quatremère de Quincy especificaba que el arte y la composición urbana, por medio de sus formas materiales, estaban haciendo posibles construcciones que expresan cualidades intelectuales e ideas morales mediante el acuerdo y la conveniencia de todas sus partes constituyentes, expresando su naturaleza, su propiedad, su uso y destino, y señalaba "Cuanto más contribuye el decoro de una ciudad a la comodidad de los habitantes, más se acerca a la perfección" (Quatremère, 1788).

Hasta cierto punto, este texto muestra que a lo largo del siglo XVIII comenzó un cierto cambio hacia la consideración del papel del ornamento en el arte urbano. Un cambio que operará durante más de un siglo a través de la formación de arquitectos Beaux Arts. Además, a comienzos del siglo XIX, los diferentes agentes que desean participar en la construcción de la ciudad ajustan sus intereses y los de una Administración cuyo objetivo primordial es el control figurativo del espacio urbano. Una base para estas operaciones es el despliegue de varias ordenanzas, reglamentos y leyes para garantizar el control figurativo del espacio (Haddad, 2017; Remesar y Ríos, 2018; Ríos, 2017; Sabaté, 1999).

Sin embargo, a lo largo del siglo XIX, y en el contexto de la introducción progresiva del modo de producción capitalista, la modernización de las ciudades se basó en los avances de la ciencia y la tecnología. Esto implicó 
un enfoque intenso en temas de higiene o salud urbana, conceptos estrechamente relacionados con la moral, como describió Engels a Manchester ${ }^{5}$. Engels señala que "sin importar cuán inconveniente sea un cobertizo, siempre habrá una persona pobre que no puede pagar una mejor, siendo la única preocupación para obtener la mayor ganancia posible" (Engels, 1845), señalando los problemas subyacentes de la inmigración, la explotación del trabajo y el deseo para el beneficio capitalista como las principales razones de esta desastrosa situación. La provisión de viviendas para las clases bajas se convierte en el principal problema que perdurará, en diferentes oleadas, a lo largo del siglo XIX hasta la actualidad. Además, al menos desde un punto de vista teórico, la cuestión del decoro adquiere nuevas dimensiones: la de la higiene (ventilación, luz solar, etc.) y la de la justicia social que tendrá un gran impacto en los procedimientos y metodologías de un nuevo campo de conocimiento, el urbanismo, que viene a reemplazar el arte urbano como instrumento de organización de la ciudad.

\footnotetext{
5 "Esa es la antigua ciudad de Manchester, y al releer mi descripción debo admitir que, lejos de ser exagerada, no he tenido las palabras adecuadas para exponer la realidad de la suciedad, la decrepitud y la incomodidad que hay allí; y cómo la construcción de este barrio, donde al menos viven entre 20 y 30 mil personas, es un desafío a todas las normas de higiene, ventilación y moral. Y tal distrito existe en el corazón de la segunda ciudad de Inglaterra, la primera ciudad industrial del mundo" (Engels, 1845)
}

Así lo hace Cerdà en su Teoría de la Construcción de Ciudades. Si bien las propuestas de Cerdà no fueron reconocidas internacionalmente, las operaciones del Barón Haussmann en París se convirtieron en un "modelo" internacional. Estas operaciones consistieron en rearticular la ciudad rompiendo el viejo tejido para permitir la conexión entre el centro y las nuevas estaciones de ferrocarril. La transformación del mercado inmobiliario parisino "upset traditional notions of community as much as they upset the sociospatial structure, and transformations in financial structures and labour processes had no less an impact upon the material basis of class relations" (Harvey, 2003, p. 219).

El paisaje parisino $y$, posteriormente, el de muchas ciudades en todo el mundo siguiendo el modelo de Haussmann, cambiará radicalmente. El equipo de Haussmann, liderado por Alphand y Davioud introducen una nueva concepción del diseño de las calles y su mobiliario cambiando la apariencia de esta capital, desde ahora caracterizada por sus jardines y bulevares,

\footnotetext{
6 "He tenido que empezar a lidiar con la influencia que el aire atmosférico, la luz del sol, el agua potable y la naturaleza de las localidades y la tierra en la que se han fundado, tienen que ver con la salud de las poblaciones. Tenía que establecer cómo entender los diferentes tipos de edificios y sus condiciones de saneamiento, moral y decencia, de independencia y economía, que nuestra civilización reclama para todos y cada uno de sus ciudadanos. Finalmente, he venido a dibujar el trazado geométrico de las calles y bloques asegurando que toda la ciudad cumpla con las condiciones higiénicas y morales en armonía con lo económico y político, para hacer factible el proyecto" (Cerdà, 1991).
} 
constituyendo lo que vine en llamar el "paradigma Alphand-Davioud-Hittorff" de la calificación de la ciudad (Haddad, 2017; Remesar, 2007). También cambió el paisaje de las formas de vida y de vivienda en París:

\section{Le hemos dado la medida de nuestro talento arquitectónico a los edificios de cinco plantas que componen el nuevo París, donde la población se apila en barracones: edificios uniformes, inconve- nientes, cuyo prototipo son los cuarteles $y$ el hotel adornado es la obra maestra" (Proudhom, 1865).}

El propio Cerdà7 critica duramente estas formas de hacer ciudades que se extienden por todo el mundo. Sin embargo, en pocas décadas, se cuestiona

\footnotetext{
7 "Hubo momentos en que la Administración Pública previó con un disfrute inocente, el apilamiento y agrupamiento de apartamentos, habitaciones y piezas, porque al mismo tiempo que restaba atención al alojamiento del exceso del vecindario, la Administración Pública podría aumentar ostensiblemente lo que se llama riqueza e impuestos públicos. Por lo tanto podemos decir que esta transformación se realizó con un aplauso general, a excepción de los inquilinos que vieron y contrastaron cómo disminuyó el bienestar doméstico y de las instalaciones a la vez que las rentas aumentaban al mismo ritmo. Además, los ornamentos públicos, la apariencia del conjunto, mejoraban admirablemente. Cada bloque (Intervias) parecía un palacio, y las calles (vías) algo más amplias y mejor alineadas, especialmente las de primer orden, las privilegiadas, aquellas que los viajeros deberían visitar, que en lugar de calles parecían paseos Y, mientras tanto, la Administración Pública, por vanidad a veces y algunas veces obedeciendo a un sentido de humanidad impulsado por higienistas, plantó árboles y construyó pequeños jardines donde tenían espacio. (...) Po tales medios, nuestras ciudades han alcanzado ese estado de magnificencia deplorable que ni satisface ni corresponde a las necesidades actuales e inspira sospechas fundadas y desconfianza para el futuro a hombres pensativos serios" (Cerdà, 1867)
}

el modelo de París ${ }^{8}$ y el sistema Beaux Arts para producir una "ciudad bella"9 porque, aunque se basó en los parámetros clásicos del "Art Urbain", comenzó a expandirse hacia un eclecticismo monumental y ornamentista. El modelo de Haussmann no había resuelto ni el problema de la vivienda ni había creado una ciudad para todos -uno de los ideales del Plan Cerdà para Barcelona- tampoco había respetado la creciente preocupación por el pasado de la ciudad. Con la ruptura del tejido histórico de la ciudad y la consiguiente desaparición de los "monumentos", el embellecimiento frontal de la ciudad presente en los bulevares parisinos requiere una corrección del modelo y las formas de abordar los problemas urbanos. De esta manera, en palabras de Sitte, cualquiera que quiera aparecer como adalid de la estética de la calle debe estar convencido, primero, de que los medios actuales para satisfacer los requisitos de tráfico son, tal vez, infalibles y, en segundo lugar, estar preparado

\footnotetext{
${ }^{8}$ Un modelo que en palabras de Harvey se puede resumir de la siguiente manera "Money, finance, and speculation became such a grand obsession with the Parisian bourgeoisie ("business is other people's money," cracked Alexandre Dumas the younger) that the bourse became a centre of corruption as well as of reckless speculation that gobbled up many a landed fortune" (Harvey, 2003, p. 118).

${ }^{9}$ En su trabajo clásico, Olsen, analizando el caso de los programas de embellecimiento en Londres, París y Viena, escribió; "The three programmes shared a number of characteristics: they resulted from the initiative of central government, depended, for their success, on the attraction of private investment by speculative builders and developers; were intended to make royal or imperial residences more prominent; created public parks; mixed public and private buildings, ecclesiastical and secular purposes, residential and commercial uses; used architecture mainly in the classical tradition; put up monuments of national, imperial, dynastic, or cultural significance; built wide streets to facilitate traffic and to serve as fashionable promenades; and combined aesthetic with social and sanitary motives." (Olsen, 1986).
} 
para demostrar que las necesidades de la vida moderna (comunicación, higiene, etc.) no son necesariamente obstáculos para el desarrollo del arte de la calle.

Precisamente en la forma de ordenar las ciudades, más que en cualquier otro aspecto, que el arte debe ejercer su influencia educativa ya que sus actividades se sienten en cada momento en el alma de la gente, y no, por ejemplo, en conciertos o espectáculos reservados para las ricas clases de la nación. Por lo tanto, sería deseable que el gobierno brindase a la estética de la calle toda la importancia que merece (Sitte, 1889).

\section{Estética de la ciudad:}

\section{diseño cívico}

La demanda de Sitte se consolidará en el nuevo siglo con la aparición de un movimiento difuso que tomará varias formas en el movimiento Art Public. A fines del siglo XIX, las ciudades enfrentaban el triple problema: un problema urbano (físico e infraestructural), un problema cívico (social, cultural y simbólico) y un problema político (vinculado al crecimiento de la democracia participativa).

Por lo tanto, no sorprende el surgimiento de conceptos como Art Public (en el área francófona), Civic Art (en los Estados Unidos) y Civic Design (en Gran Bretaña) como una forma empírica y teórica para pensar y resolver la organización de la ciudad que está comenzando su expansión a escala metropolitana. Estos conceptos giran en torno a la idea de la necesidad de una "estética cívica".

A primera vista, dando a este estudio el título de estética de la ciudad, parece que subordinamos todo a la belleza, [pero ...] ya argumenté que los trabajadores del arte industrial serían capaces de desarrollar, en perfecta armonía, la forma y el uso del objeto'o (Buls, 1893).

La actividad de Buls como alcalde de Bruselas facilita el surgimiento de lo que ahora llamamos un "think-tank" centrado en el problema de la estética urbana ${ }^{11}$. Un breve análisis de esta corriente servirá para aclarar el pensamiento sobre el decoro urbano. Ya se ha señalado que, en el siglo XIX, el concepto del decoro urbano se había ampliado hacia aspectos sociales y económicos, implícitos en el paradigma higienista que se cruzaba, de

\footnotetext{
${ }^{10}$ Buls añade "(...) Pero los funcionarios de una gran ciudad que tiene una historia y que conserva restos, jay! demasiado raros del pasado, no deben considerar sólo las instalaciones de movimiento. Deben recordar que pertenecen a una nación, que cuenta en la historia del arte y en la que los ciudadanos se enorgullecen de adornar la ciudad de su nacimiento. Ahora sucede que, si uno busca reglas estéticas aplicables a ciudades antiguas con la idea de cambiarlas según lo requerido por la vida moderna, es precisamente siguiendo los principios indicados por el ingeniero que el artista encontrará las soluciones más cercanas a su ideal. No nos cuenten, por lo tanto, entre los admiradores de la conservación del pasado, quienes, amantes exclusivos de lo pintoresco, lamentan la bóveda del Sena y suspiran por las ruinas infectadas que solían engendrar fiebres en el torrente de aguas sucias". (Buls, 1893).

${ }^{11}$ Mediante la Oeuvre Belge d'Art Public (Broerman, 1898); (Abreu, 2006). Un análisis del trabajo relacionado con los Congresos Internacionales de Arte Público organizados por el Oeuvre Belge permite definir cuál fue la idea subyacente, no solo limitada a las ciudades europeas (Crouch, 1915; Monclús, 1995) sino con un gran impacto en las ciudades de América del Norte (De W., BC, 1900; Robinson, 1904; Hegemann \& Peets, 1922).
} 
un modo u otro, la totalidad del pensamiento sobre la ciudad.

Ahora, la idea de "Arte público - Arte cívico - Diseño cívico" amplía la base de este concepto a otras dimensiones, incluida la justicia social y el atractivo necesario de las ciudades para el turismo. Además, el tema recurrente de la mejora de la ciudad mediante el arte, especialmente por medio de su embellecimiento, en las conferencias internacionales dedicadas al tema, resaltan la necesidad de: defensa de los sitios históricos y del patrimonio artístico; defensa de la cultura popular; defensa de las ciudades jardín; y, más prominentemente, el desarrollo de la educación artística. Todo esto en el contexto de proporcionar vivienda a las clases sociales desfavorecidas.

Es preciso señalar que, en Francia, el concepto de arte público, se vincula al surgimiento del Museo Social' ${ }^{12}$.

\footnotetext{
12 "The Social Museum was founded in 1894, but more firmly rooted earlier in the social economy section of the 1889 Universal Exhibition in Paris, the Musée social was a republican think-tank that brought together reformers from diverse social, political, and ideological backgrounds. As such, it represented the vibrant para political sphere that helped shape the debate on social welfare, for the Musée social operated not only as an institution for social research, but it also maintained an immense library, published reports of Musée-sponsored studies, hosted public lectures on diverse topics, and provided consultation services for those interested in sponsoring reform programs in their own companies or creating new self-help associations like mutual aid societies. In fact, the Musée social's reputation for expertise in social welfare and vigorous debate on all facets of the social question was enshrined in its unofficial title, "the antechamber of the Chamber." Virtually every piece of socia legislation proposed between 1895 and 1920 had received ample scrutiny at the Musée before being presented to French legislators. Even the deputé Cornudet admitted that the 1919 urban planning law that bears his name was drafted within the halls of the Musée social because of its focus on public hygiene" (Beaudoin, 2003)
}

Bajo el paraguas del Museo Social en 1905, impulsado por la Asociación General de Ingenieros Municipales, Arquitectos e Higienistas, se crea la Sección de Higiene Urbana y Rural. En 1910, varios miembros de esta Sección asisten a la Conferencia Internacional sobre Urbanismo (Londres) y participan en el concurso internacional para el Gross Berlin. Poco después, fundaron la Sociedad Francesa de Arquitectos y Planificadores (SFAU) con la participación de personajes como Agache, Auburtin, Bérard, Hébrard, Forestier, Jaussely, Parenty Prost o Redont, Eugène Hénard era su presidente.

Como se señaló anteriormente, los miembros de esta Sección desarrollan estudios y proyectos urbanos para las ciudades francesas, pero también para varias ciudades europeas y estadounidenses, estableciendo relaciones con los urbanistas británicos, estadounidenses y europeos. Como sucederá más adelante con el Museo Social de Barcelona ${ }^{13}$ (1909),

\footnotetext{
${ }^{13}$ Un claro ejemplo de esta duplicidad sería el enfrentamiento de Barcelona, por un lado, con el problema de la Reforma Interior del área histórica y, por otro, con la expansión de la ciudad a escala metropolitana. Refiriéndose a la reforma, Puig i Cadafalch, uno de los mayores críticos de Cerdà y más tarde presidente de la Mancomunidad de Cataluña afirmó: "Debemos estudiar la reforma desde un punto de vista artístico (...). Es necesario hacer lo que Buls hizo en Bruselas con la Grand Place: no para destruir, sino para reconstruir. devolviendo las cosas a su primitiva belleza" (Puig, 1900, 1901a, 1901b). Mientras tanto, en 1903, el Ayuntamiento lanzó una convocatoria internacional, "Concurso internacional de anteproyectos para vincular el área del Eixample de Barcelona, las ciudades agregadas y el resto de los municipios de Sarria y Horta". Leon Jaussely, destacado representante de la tradición del Art Public francés, ganó este concurso (Fiol, 2008).
} 
los miembros de esta Sección están vívidamente influenciados por las propuestas de Ebenezer Howard, Parker y Unwin sobre la ciudad jardín, pero también por la idea belga de Art Public.

El Museo Social, a través de su Departamento de Higiene Rural y Urbana, alberga la creación, en 1917, de la Ecole d'Art Public. Esta escuela es una iniciativa de Marcel Poëte que, en 1904, fundó la Bibliothèque des Travaux Historiques de París. Esta escuela publica la revista L’Art Public. Louis Bonnier (1917) señaló, en el primer número de la revista, que la escuela está organizada desde una perspectiva multidisciplinaria, posiblemente inspirada en su contraparte en Liverpool. En 1909, William Lever, patrocinador del suburbio jardín industrial en Port Sunlight, cerca de Liverpool, estableció un Departamento de Diseño Cívico en la Universidad de Liverpool con el fin de capacitar a arquitectos, inspectores e ingenieros en la nueva disciplina del Town Planning y creó la Town Planning Review bajo la dirección del joven Patrick Abercrombie. En 1919, tras la creación de la Sociedad Francesa de Urbanistas, la Escuela de Arte Público se convertirá en la Escuela de Estudios Urbanos que, en 1924, se convertirá en el Instituto de Planificación Urbana de la Universidad de París.

En el siglo XIX, fuimos testigos del aumento en lo que se denominó la "clase ociosa" (Veblen, 1899) y de lo que Baudelaire (1859-1863) etiquetó con los términos "hombre del mundo", dandy y "flâneur".
Baudelaire issued his manifesto for the visual arts (and a century before Benjamin attempted to unravel the myths of modernity in his unfinished Paris Arcades project). Balzac had already placed the myths of modernity under the microscope and used the figure of the flaneur to do it. And Paris-a capital city being shaped by bourgeois power into a city of capital - was at the centre of his world (Harvey, 2003, p. 24).

Los responsables de este crecimiento fueron: el incremento de la actividad económica desde los procesos de industrialización, la internacionalización económica y el desarrollo de los sistemas de transporte (ferrocarril, barcos, etc.), generando un nuevo interés social que viene a reemplazar al Gran Tour de anteriores edades: el turismo. El surgimiento gradual de una clase con tiempo de ocio y recursos económicos disponibles, en paralelo a un conjunto de actividades relacionadas orientadas a conocer el mundo entero (las exploraciones, por ejemplo), implican que el mundo puede ser conocido en un solo lugar: el sitio de una "exposición internacional". José Martí, el apóstol de la libertad cubana y editor de $L a$ edad de oro, escribió en el exilio en Nueva York: "Y eso lo veremos ahora, como si lo tuviéramos frente a nuestros ojos. Vamos a la exposición; todas las razas humanas están haciendo esta visita. Veremos en el mismo jardín árboles de todos los pueblos de la Tierra" (Martí, 1889, p. 1).

Desde su creación en Londres (la Gran Exposición, 1851), siguen uno tras otro hasta ahora. El BIE (Bureau of International Expositions) estima que entre 1851 (Londres) y 1900 (París) el número total de visitantes 
superó los 188 millones de personas, sin contar la gran cantidad de personas que viajaron a ferias internacionales, regionales o locales. Este turismo, cada vez más masivo, necesita soluciones materiales para alojamiento $^{14}$ y transporte y organización del tiempo. Sobre todo, el turismo requiere del capital simbólico que Bourdieu llamó capital cultural objetivizado: información, visitas guiadas, guías turísticas, incluyendo elecciones previas de sitios, de edificios, de paisajes (Bourdieu, 1988): ¿Cuáles? ¿Cómo seleccionarlos? ¿Quién elegirá? ¿Por qué estas opciones y otras no? Emergen entonces instituciones, a menudo municipales, dedicadas al desarrollo del turismo en una ciudad o provincia (en francés Syndicats d'Inititatives). El turismo agrava el problema entre lo nuevo y lo viejo. Un problema de ciudadanía, la construcción de una nueva ciudad enfrenta la necesidad de preservar algo de su pasado, sus monumentos.

Una ciudad próspera debe inevitablemente transformarse para adaptarse a las nuevas necesidades de movimiento, limpieza, higiene y comodidad. No puede, sin embargo, descuidar las calificaciones morales e intelectuales de una ciudad vigilada que conserva en sus monumentos huellas del pasado, gloriosas memorias históricas, artísticas y poéticas (Buls, 1893).

\footnotetext{
14 Un ejemplo poco conocido pero muy interesante es el Hotel International que Domènech i Montaner construyó para la Exposición Internacional de Barcelona de 1888. Utilizando la racionalidad constructiva que ofrece el hierro fundido, Domènech construyó, en 60 días, el hotel con capacidad para dos mil huéspedes, con 600 habitaciones y 30 apartamentos para familias numerosas.
}

Refiriéndose a Barcelona, Martorell, señala la influencia de los movimientos relacionados con el arte público y la estética urbana al plantear que en la ciudad

se están haciendo esfuerzos para vincular el aspecto pintoresco, montañoso y monumental presente en las ciudades antiguas, al tiempo que sirven a la higiene y las necesidades actuales del tráfico diario y las comunicaciones. Existe un gran arte con sus obras, sus escuelas, sus maestros y su literatura. Nombres como Stübben, Henrici, Sitte o Buls son hombres eminentes que han desarrollado este arte, principalmente en Alemania, Austria, Bélgica al principio, y luego en Italia, Inglaterra y los Estados Unidos (Martorell, 1911).

Martorell proclama esta situación al inicio de las obras de la Vía Layetana en Barcelona en la que se asiste a la invención del "Barrio Gótico"15, uno de los destinos turísticos de la ciudad. Sin embargo, ¿existe realmente el Barrio Gótico? Desde principios de los años 20 , las voces en contra de esta denominación se elevan, ya que, desde un punto de vista histórico y arqueológico es difícil conceder o ubicar la emergencia de éste, en ese contexto en el que lo "Gótico" hizo su aparición en la historia de Barcelona, en lo estético, estilístico, estructural u organizacional de la ciudad.

\footnotetext{
15 "El Barrio Gótico tal como lo concebimos sería como una preciosa caja que guardara las deliciosas joyas de Barcelona: la Catedral y el Palacio de los Reyes. Todas las calles incluidas en su perímetro deberían regresar, no al estado primitivo de la época histórica cuando se completaron, sino al estilo gótico catalán, gracias a la mano experta y sabia de los mejores arquitectos modernos de Cataluña. Las fachadas de las casas y, si es posible, las propias casas, deberían adaptarse con la máxima pureza a las reglas góticas, desarrollando, con la debida sobriedad, un ambiente de barrio, mediante los recursos fantásticos que han venido de los antiguos constructores catalanes de la época. Y una maravillosa unidad florecería en ese recinto, que sería como el corazón de la ciudad de Barcelona, cuidadosamente conservado en un relicario" (Rucabado, 1911).
} 


\section{Conclusiones}

Estos apuntes, como sugiere el título, pretenden hacer una reflexión no sobre la historia sino sobre el presente de nuestras ciudades, ciertamente a la luz del saber generado con los años. Hoy, al igual que hace cien años, nuestras ciudades padecen de algunos conflictos urbanos que guardan cierta relación con aquellos que pretendieron solucionar los urbanistas y diseñadores urbanos: saturación urbana, problemas de transporte y vialidad, especulación inmobiliaria y presión del turismo. La mejora de los espacios públicos con la intención de crear ambientes urbanos de calidad precisa re-pensar el papel que el Arte, concretamente el Arte Público, y el Diseño Urbano pueden tener en la generación de ciudades más habitables, obviamente, sin abandonar los principios multiescaleres, sostenibles y de innovación urbana como modo de lucha contra la segregación social y que deberían definir las políticas urbanas para el siglo XXI. Revisando el pasado podemos aprender de sus aciertos y de sus errores.

\section{REFERENCIAS}

\section{Abreu, J. (2006). Escultura Pública e Monumentalidade em Portugal (1948-1998). [Tesis]. Unviersi- dade Nova de Lisboa, Lisboa. Recuperado de https://run.unl.pt/ bitstream/10362/31390/1/Escultura Publica_e_Monumentalidade_em_P. pdf}

Alberti, L. (1992). De Re Aedificatoria (1452). Edición española a cargo de Javier Fresnillo. Madrid: Ed. Akal.

Beaudoin, S. (2003). A Social Laboratory for Modern France: The Musee Social and the Rise of the Welfare State. J. Soc. Hist. 37, 560-562. https://doi. org/10.1353/jsh.2003.0166

Bonnier, L. (1917). Pourquoi nous ouvrons une Ecole d'Art Public? L'Art Public, 1(1). 18

Bourdieu, P. (1988). La distinción. Criterio $y$ bases sociales del gusto. Madrid: Taurus.

Broerman, E. (1898). Historique de Lœuvre de l'art public. In, A. Benard, Oeuvre de l'Art Public. Premier Congrés International. Bruxellles, Belgique.

Buls, C. (1893). Esthétique des villes. Brussels: Bruylant-Christophe \& Cie.

Cerdà, I. (1867). Teoría general de la urbanización y aplicación de sus principios y doctrinas a la reforma $y$ ensanche de Bacelona. Madrid: Imprenta Española.

Cerdà, I. (1991). Teoría de la construcción de las ciudades: Cerdà y Barcelona. En: Teoría de la construcción de las ciudades aplicada al proyecto de Reforma y Ensanche de Barcelona (1859). Madrid, Barcelona: Ministerio para las Administraciones Públicas, Secretaría General Técnica, Ajuntament de Barcelona. 
Choay, F. (1998). Dictionnaire de l'Urbanisme et de l'aménagement. París: Presses universitaires de France.

Consejo Europeo de Urbanistas-CEU. (1998). Nueva Carta de Atenas. Atenas: CEU. Recuperado de http://www. unesco.org/culture/natlaws/media/pdf/ guatemala/guatemala_nueva_carta_ atenas_1998_spa_orof.pdf

Crouch, Ch, (1915). Design Culture in Liverpool 1880-1914. The Origins of the Liverpool School of Architecture, 2002nd ed. Liverpool: Liverpool University Press.

Engels, F. (1845). La situación de la clase obrera en Inglaterra. Buenos Aires: Editorial Futuro.

Fiol, C. (2008). Retícules i diagonals el Pla Jaussely de Barcelona de 1907 $i$ el Pla Burnham de Chicago de 1909. [Tesis].Universitat Politècnica de Catalunya, Barcelona. Recuperado de https://upcommons.upc.edu/ handle/2117/94281

Gaston, (2014). Decorum (Grove Dictionary of Art). In, The rhetoric of art: Varietas, empathy and decorum. Recuperado de http://www2.idehist.uu.se/distans/ ilmh/Ren/password-decorum.htm

Giedion, S. (1952). Historical Background to the Core. J., Tyrwhitt, J., Sert \& E., Rogers (Eds.). The Heart of the City. Towards the Humanisation of Urban Life. (17-25). London: Lund Humphries \& Co Ltd.
Haddad, M. (2017). The Eventrement of Paris and the configuration of the Alphand-Davioud-Hittorff Paradigm on urban design. On the w@terfront, 51(1). 25-49.

Harvey, D. (2003). Paris, Capital of Modernity. New york: Routledge.

Hegemann, W. \& Peets, E. (1922). The American Vitruvius: An Architect's Handbook of Civic Art. New York: Architectural Book Publishing Co.

Kahn, A. (2002). Practices, Pedagogies, Premises. In, Van Allen Institute, Urban design now 12. Van Allen Institute, New York.

Kohane, P., \& Hill, M. (2001). The eclipse of a commonplace idea: decorum in architectural theory. Arq Archit. Res. Q. 5(1), 63-77. https://doi.org/10.1017/ S1359135501001105

Mandanipour, A. (2007). Designing the City of Reason. Foundations an Frameworks. New york-london: Routledge.

Martí, J. (1889). La Exposición de París. La Habana: Centro de Estudios Martinianos.

Martorell, G. (1911). La Urbanisació moderna. La llustració Catalana $2^{\circ}$ época, 10-12.

Monclús, F. (1995). Arte Urbano y Estudios Histórico-Urbanísticos, 3ZU. Tradiciones, ciclos y recuperaciones. Revista de Arquitectura (ETSABAmbit), 4. 92-101. 
Olsen, D. (1986). The City as a Work of Art. London-Paris-Viena: Yale University Press.

Proudhom, P-J. (1865). Du principe de l'art el de la destination sociale. Paris: Garnier.

Puig, J. (1900). Barcelona d'anys á venir I. La Veu de Catalunya, 1, 1.

Puig, J. (1901a). Barcelona d'anys á venir II. La Veu de Catalunya, 2, 1.

Puig, J. (1901b). Barcelona d'anys a venir III i últim. La Veu de Catalunya, 2, 1.

Quatremère, A-Ch. (1788). Encyclopédie méthodique: Architecture, AntoineChrysostome Quatremère de Quincy. Paris: chez Panckoucke libraire.

Remesar, A. (2007). O "estilo AlphandDavioud- Hittorf" de mobiliário urbano nos centros históricos. O journal negócios do mercado das cidades, 28(1). 8-9.

Remesar, A. y Ríos, M. (2018). Barcelona s. XVIII, un paisatge en construcció. On the w@terfront, 60(1). 9-77.

Ríos, M. (2017). ¿Hacer Ciudad? Barcelona, la construcción del paisaje, 1929 -1973 [Doctoral]. Universitat de Barcelona, Barcelona.
Robinson, Ch. (1904). Modern civic art or The city made beautiful. New York: Putnam and Son.

Rucabado, R. (1911). Un barrio gótico en Barcelona. La Cataluña, 189(1). 309-311.

Sabaté, J. (1999). El proyecto de la calle sin nombre. Los reglamentos urbanos de la edificación ParísBarcelona. Barcelona: Fundación Caja de Arquitectos.

Sert, J. (1957). The Human Scale. Key to the measure of cities. In, E., Mumford, (Ed.), The Writings of Josep Lluís Sert. (185). Yale University PressHarvard Graduate School of Design, New Have and London, Cambridge (Mass).

Sitte, C. (1889). City Planning According to Artistic Principles. London: Phaidon Press

Tafuri, M. (1968). Teoria e Storia dell' architettura. Roma: Laterza.

Veblen, T. (1899). The Theory of the Leisure Class An Economic Study of Institutions. México, D.F.: Fondo de Cultura Económica. 